\title{
Sealing the Gap of Vertical Root Fracture through the Root Canal
}

\author{
Tsutomu Sugaya $^{1 *}$, Megumi Natatsuka', Youji Motoki', Kana Inoue ${ }^{1}$, Saori Tanaka', Hirofumi Miyaji', Masamitsu Kawanami' and Ryuji \\ Sakagami ${ }^{2}$
}

${ }^{1}$ Department of Periodontology and Endodontology, Hokkaido University School of Dental Medicine, Sapporo, Hokkaido, Japan

${ }^{2}$ Department of Periodontology, Fukuoka Dental College, Fukuoka, Japan

\begin{abstract}
Objective: The objective of this retrospective study was to investigate whether preparing the fracture line through the root canal before bonding a vertically fractured root would result in improvement of periodontal inflammation.

Methods: A total of 83 teeth from 81 patients, who had been diagnosed with vertically fractured roots, and in whom the fracture gap was sealed through the root canal, were used. The fracture line was prepared using an ultrasonic tip under a microscope in the preparation group $(n=60)$ and 4-META/MMA-TBB resin was used to bond the fractured gap. In the nonpreparation group $(n=23)$, the fracture line was not prepared. Reevaluation was done 1 to 2 months later. Patients were clinically examined for probing depth and presence or absence of sinus tract and abscesses, and radiographs were taken to gauge improvement of bone defects.
\end{abstract}

Results: Comparing the probing depth in the non-preparation group before and after treatment, there was no significant difference $(p=0.13)$, but in the preparation group, the probing depth was significantly shallower following treatment $(p<0.001)$. In teeth where pre-treatment bone defect occurred, disappearance or decrease of bone defect was seen in 8 out of 13 teeth $(61.5 \%)$ in the non-preparation group and 33 out of 41 teeth $(80.5 \%)$ in the preparation group. There was significant improvement in the preparation group compared with non-preparation group $(p=0.008)$.

Conclusion: The results suggest that preparing the fracture line through the root canal before sealing a vertically fractured root may be effective in resolving periodontal inflammation.

Keywords: Vertical root fracture; Gap sealing; Preparing the fracture line; Adhesive resin; Periodontal inflammation

\section{Introduction}

A vertical root fracture may lead to rapid destruction of periodontal tissue. Generally, if the tooth is single-rooted, the tooth is extracted, and if it has multiple roots, the root involved is resected or hemisection is performed [1-4]. A number of studies have described efforts to clinically preserve teeth, with particular success reported with resin bonding [5-9]. Sugaya et al. used 4-methacryloxyethyl trimellitate anhydride/methyl methacrylate tri-n-butyl borane (4META/MMA-TBB) resin to bond 23 teeth with vertically fractured roots, performing bonding either through the root canal or with the intentional replantation method, and reported after 6 to 74 months of observation that $18(78 \%)$ of the teeth could be preserved [5]. Hayashi et al. extracted 26 teeth with vertically fractured roots, bonded the fractured roots and replanted them [8]. They reported that, after 36 months, it had been possible to preserve $69.2 \%$ of the teeth. The results described in these reports suggest that bonding is effective for vertically fractured tooth roots. However, it has been pointed out that bonding through the root canal more likely results in insufficient improvement in periodontal pockets and bone defects than intentional replantation [5]. This might be explained by the presence of residual bacteria in the fracture gap, and the fact that it may be more difficult to completely bond the fracture gap when bonding through the root canal.

At the same time, Kimura et al. fractured roots of beagles vertically and performed histopathological evaluation of the periodontal tissue [10]. They found that periodontal inflammation was caused primarily by bacteria proliferating in the root canal and fracture gap. There was only slight down growth of pocket epithelium and the periodontal probe only penetrated the inflammatory connective tissue. Tomita et al. on the other hand, reported that by sealing the fracture gap, the probing depth becomes shallow, and bone defects in the area around the fracture line heal [11]. In this experiment, bonding the fracture gap with resin through the root canal resulted in incomplete sealing in many cases and periodontal inflammation. Given this, it is believed that inflammation of periodontal tissue is more likely to improve if the fracture line is prepared through the root canal, infected substance is removed from the fracture gap, and a space is created into which the resin can penetrate.

The objective of the present study was to clinically investigate whether preparing the fracture line through the root canal before bonding a vertically fractured root would result in improvement of periodontal inflammation.

\section{Materials and Methods}

This retrospective study was carried out in teeth diagnosed with vertical root fracture and in which the fracture gap was sealed through the root canal at the Department of Endodontics and Periodontics, Hokkaido University Hospital between 1994 and 2012. The protocol of this study was approved by the Institutional Review Board of Hokkaido University Hospital for Clinical Research. Examination items included the patients' age, sex, the general condition of the patient, type of tooth, the initial condition of pulp tissue, fracture site, fracture region in longitudinal axial direction and probing depth before and after treatment, as well as the status of bone defects as indicated on dental $\mathrm{X}$-rays (Figure 1A), and whether or not the fracture line had been prepared through the root canal. If there were fracture lines in two sites in a single tooth, the deeper of the two probing depths was used as the site for examination.

*Corresponding author: Tsutomu Sugaya, DDS, PhD, Associate Professor, Department of Periodontology and Endodontology, Hokkaido University School of Dental Medicine, N13 W7 Kita-ku, Sapporo, Hokkaido 060-8586, Japan, Tel: +8111-706-4266; Fax: +81-11-706-4334; E-mail: sugaya@den.hokudai.ac.jp

Received December 17, 2015; Accepted January 08, 2016; Published January 16,2016

Citation: Sugaya T, Natatsuka M, Motoki Y, Inoue K, Tanaka S, et al. (2016) Sealing the Gap of Vertical Root Fracture through the Root Canal. Dentistry 6: 354 doi:10.4172/2161-1122.1000354

Copyright: $\odot 2016$ Sugaya T, et al. This is an open-access article distributed under the terms of the Creative Commons Attribution License, which permits unrestricted use, distribution, and reproduction in any medium, provided the original author and source are credited. 


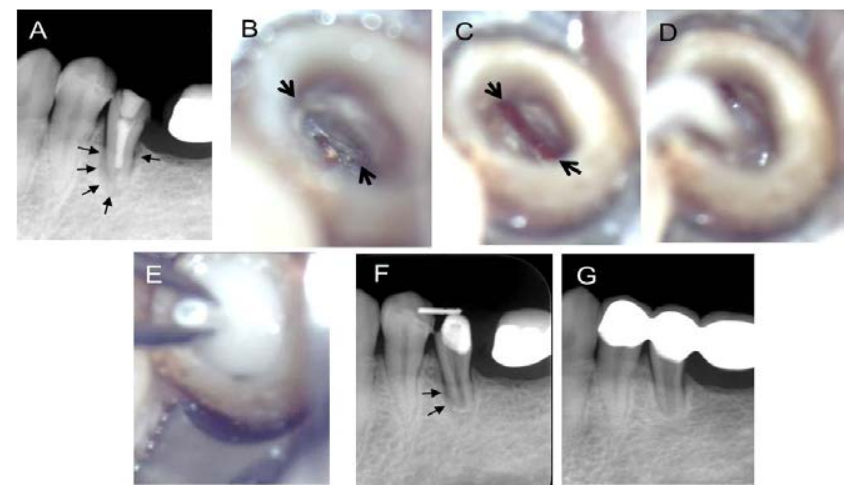

A: Radiograph taken at initial visit. Bone defects (arrows) can be seen in the mandibular left premolar; B: The fracture line (black arrows) can be seen when root canal filling materials were removed; C: After preparation of the fracture line (arrows); D: Super Bond was injected inside the root canal with a syringe; E: Insertion of a fiber pos; F: Two months later, decrease of bone defects (arrows); G: One year later, the bone defect had disappeared.

Figure 1: A case of apical region fracture in which the fracture line was prepared through the root canal (44-year-old woman)

Diagnosis of vertically fractured roots was performed under microscope from inside the root canal, after removing the post, root canal filling material, and infected substance (Figure 1B). The site of fracture was classified into buccal and/or lingual, mesial and/or distal, and other (fracture affecting both buccal/lingual and mesial/distal sites). The fracture regions in longitudinal axial direction were classified into complete fractures or incomplete fractures, and incomplete fractures were further classified into the three subgroups of cervical region fracture, mid-region fracture, and apical region fracture [12].

The exclusion criteria of the study were as follows: the fracture gap was already wide, granulation tissue had penetrated into the fracture gap, and the fracture line could not be verified because of root curvature.

Roots were divided into two groups, depending on the treatment method; a preparation group, and a non- preparation group. In the preparation group, initially fracture lines that could be confirmed under a microscope (Mani scope Z, Mani, Tochigi, Japan) were prepared for all cases through the root canal until the periodontal ligament was reached (Figure 1D). Preparation was performed using an ultrasonic device (ENAC 10WA, Osada, Tokyo, Japan) and an ultrasonic tip (SC4-17, Osada, Tokyo, Japan) with a point diameter of $0.25 \mathrm{~mm}$, a 0.05 taper, and a length of $17 \mathrm{~mm}$ for all cases in the preparation group. It was performed without irrigation, with irrigation occasionally performed when ultrasonic cleaning was performed for physical debridement. At the point when bleeding began, it was judged that the tip had reached the periodontal ligament, and preparation of that site was stopped. Preparation was performed in such a way that the periodontal ligament was reached along the entire fracture line (Figure 1C). After preparation had been completed, if bleeding and pus discharge from the fracture gap did not stop, calcium hydroxide was applied and the root canal was temporarily sealed. During the followup visit, the root canal was thoroughly cleaned using an ultrasonic device (SC4-17, ENAC 10WA, Osada, Tokyo, Japan), and if there was no pus discharge or bleeding from the fracture gap, the fracture gap and post were bonded in the next procedure. The root canal was filled with $10 \%$ citric acid with $3 \%$ ferric chloride (Green Activator, Sun Medical, Shiga, Japan) using a syringe and the surface of the dentin was treated. After 10 seconds, a three-way syringe with a $24 \mathrm{G}$ cleansing needle connected was used to wash the root canal. Water in the root canal was then suctioned, and the canal was dried by mild air blow using a threeway syringe with a $24 \mathrm{G}$ cleansing needle. 4-META/MMA-TBB resin (Super Bond, Sun Medical, Shiga, Japan) was mixed with monomer, a catalyst, and polymer according to the manufacturer's instructions, and the mixture was loaded into a syringe (Terumo, Tokyo, Japan) and injected into the root canal for all cases (Figure 1D). In cases where the indirect method was used, a cast post or fiber post (i-TFC fiber, Sun Medical, Shiga, Japan) with a resin core was inserted while the fluidity of the Super Bond was still high, while in cases where the direct method was used, fiber posts were inserted into the root canal filled with Super Bond (Figure 1E), and a core was prepared using composite resin (i-TFC Core Resin, Sun Medical, Shiga, Japan).

In the non-preparation group, the fracture line was not prepared, but other treatment was the same as that for the preparation group.

In both groups for which post bonding had been performed, reevaluation of the root was done 1 to 2 months later. Patients were clinically examined for probing depth and presence or absence of sinus tract and abscesses, and radiographs were taken to gauge improvement of bone defects (Figure $1 \mathrm{~F}$ and $1 \mathrm{G}$ ). Improvement of bone defects was classified into disappearance of bone defect, decrease of bone defect and no change or increase of bone defect.

Statistical analysis was conducted using SPSS Statistics Version 21 (IBM, Armonk, NY, USA), and a Chi-square test, Mann-Whitney U test and Wilcoxon signed-rank test were performed.

\section{Results}

The patients in the non-preparation group comprised of 7 men and 16 women aged $55.7 \pm 13.3$ years (Mean \pm SD). The preparation group comprised of 19 men and 39 women aged $53.7 \pm 9.7$ years. None of the patients had general conditions or systemic diseases influencing the treatment. For both groups, premolars comprised the largest number of teeth, with no significant differences between the two groups $(\mathrm{p}>0.05)$ (Table 1). One of the teeth was vital, one was non-vital but without caries, and 21 had received root canal therapy in the non-preparation group. The corresponding numbers in the preparation group were 1 , 1 and 58 respectively, with no significant differences between the two groups ( $p>0.05$ ). Good oral hygiene was maintained in both groups.

In a comparison of fracture region in longitudinal axial direction, the percentage of apical region fracture was slightly larger for the nonpreparation group than for the preparation group, but there was no significant difference between the two groups ( $\mathrm{p}=\mathrm{p}>0.05$ ) (Table 2).

\begin{tabular}{|c|c|c|c|c|c|c|c|c|c|}
\hline \multirow{2}{*}{ Group } & \multicolumn{4}{|c|}{ Maxilla } & \multicolumn{4}{|c|}{ Mandible } & \multirow{2}{*}{ Total } \\
\hline & Incisor & Cuspid & Premolar & Molar & Incisor & Cuspid & Premolar & Molar & \\
\hline Non-preparation group & 2 & 4 & 7 & 2 & 0 & 1 & 6 & 1 & 23 \\
\hline Preparation group & 7 & 1 & 19 & 9 & 0 & 2 & 10 & 12 & 60 \\
\hline Total & 9 & 5 & 26 & 11 & 0 & 3 & 16 & 13 & 83 \\
\hline
\end{tabular}

No significant differences between groups (Chi-square test, $p>0.05$ ) 


\begin{tabular}{|c|c|c|c|c|c|}
\hline \multirow{2}{*}{ Group } & \multirow{2}{*}{ Complete } & \multicolumn{3}{|c|}{ Incomplete Fracture } & \multirow{2}{*}{ Total } \\
\cline { 3 - 5 } & $\begin{array}{c}\text { Apical } \\
\text { region }\end{array}$ & Mid-region & $\begin{array}{c}\text { Cervical } \\
\text { region }\end{array}$ & \\
\hline Non-preparation group & 5 & 10 & 1 & 7 & 23 \\
\hline Preparation group & 19 & 18 & 1 & 22 & 60 \\
\hline Total & 24 & 28 & 2 & 29 & 83 \\
\hline
\end{tabular}

No significant differences between groups (Chi-square test, $\mathrm{p}>0.05$ )

Table 2: Fracture region in longitudinal axial direction.

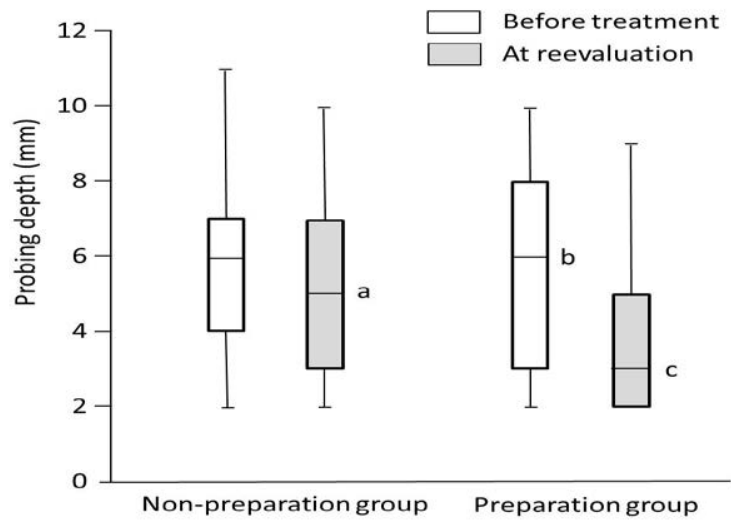

Significant difference between a and $c$ (Mann-Whitney $U$ test, $p<0.05)$; Significant difference between $b$ and $c$ (Wilcoxon signed-rank test, $p<0.01$ )

Figure 2: Box plot of probing depth before treatment and at reevaluation.

There was no significant difference between the two groups in terms of pre-treatment probing depth $(p>0.05)$, but at reevaluation, the probing depth was significantly shallower for the preparation group than the non-preparation group $(\mathrm{p}<0.05)$. Comparing the probing depth in the non-preparation group before and after treatment, there was no significant difference ( $p>0.05)$, but in the preparation group, the probing depth was significantly shallower following treatment $(\mathrm{p}<0.01)$ (Figure 2).

Radiographic examination showed bone defects prior to treatment in 13 of 23 teeth in the non-preparation group (56.5\%) and in 41 of 60 teeth in the preparation group (68.3\%), with no significant difference between the two groups $(p>0.05)$. In teeth where pretreatment bone defect occurred, disappearance or decrease of bone defect was seen in 8 out of 13 teeth $(61.5 \%)$ in the non-preparation group and 33 out of 41 teeth $(80.5 \%)$ in the preparation group. There was significant improvement in the preparation group compared with non-preparation group $(\mathrm{p}<0.01)($ Table 3$)$. In teeth that had no bone defects prior to treatment, bone defects developed after treatment in one of 10 teeth in the non-preparation group and three of 19 teeth in the preparation group, with no significant difference between the two groups $(p>0.05)$.

\section{Discussion}

Cases in which the fracture gap had widened and granulation tissue had already penetrated at the point when a diagnosis of vertical fracture was confirmed were excluded from this study. Granulation tissue may hinder the removal of infected dentin in the fracture line through the root canal, and granulation tissue, exudate, and bleeding make it difficult to achieve proper bonding of the resin when sealing the fracture gap.

The fracture line was prepared using an ultrasonic tip with a point diameter of $0.25 \mathrm{~mm}$ and a length of $17 \mathrm{~mm}$ without irrigation because irrigation water and the head of the rotary instruments such as air turbines can interfere with the field of view, so it is not possible to prepare while viewing the fracture line under a microscope. After the fracture line was prepared, the patient was excluded from this treatment method if bleeding and pus discharge into the fracture gap continued even after calcium hydroxide was applied, and there was no further improvement.

Super Bond was used for bonding the fracture gap. This resin was chosen because of reports stating that inflammation of periodontal ligament did not occur histopathologically after bonding of the fracture gap with Super Bond [11,13], and also because of a number of clinical reports [5-9] describing good outcomes when Super Bond was used to treat vertically fractured roots. Super Bond uses tri-n-butyl borane as a polymerization initiator, so when it comes in contact with moisture, radicals are generated and polymerization progresses [14]. As a result, even if moisture is present, it is still possible to obtain a high rate of polymerization and good biocompatibility.

In the preparation group, there was significant improvement of the probing depth and bone defects compared to the non-preparation group. There may be several reasons for this: it was possible to eliminate bacteria from the fracture gap by preparation; it was possible to thoroughly wash and dry the fracture gap in order to perform bonding; and Super Bond was able to fill the fracture gap more reliably. The Super Bond mixture had high fluidity and the teeth showed no percussion pain and gingival swelling after sealing the fracture gap. This may indicate that the resin did not overflow into the periodontal tissue beyond the apex or the fracture gap. The improvement in probing depth was indicated by the absence of gingival recession, which meant gain of periodontal attachment and/or reduction of penetration of pocket probe. However, in nonsurgical treatment, the possibility of attachment gain during a short period of time is small. The periodontal probe can easily penetrate into the inflamed connective tissue in marginal periodontitis [15-17]. Consequently, the probe penetrated tissue because there was inflammation of the periodontal tissue along the fracture line before treatment, but as the inflammation improved following treatment, the probe no longer penetrated tissue.

The inflammation disappeared and bone was repaired along with resolution of the fracture line infection because there was no down growth of the pocket epithelium in cases in which bone defects had improved as a result of the treatment. This is consistent with reports of animal experiments in which, if only a short time has passed since vertical root fracture, there is very little downgrowth of the epithelium, and if the fracture gap is successfully sealed, bone defects will undergo repair [11].

However, there were cases in the preparation group where no improvement in probing depth or bone defect at reevaluation was seen. These findings were probably due to incomplete sealing due to limitations in fracture line preparation accuracy, exudate and bleeding into the fracture gap that inhibited bonding and technical errors during bonding. Biofilm may have also formed on the outer root surface around the fracture line, as it does in the cementum surface around the apical foramen in apical periodontitis [18-24]. In such cases there are

\begin{tabular}{|c|c|c|c|c|}
\hline Group & Disappearance & Decrease & No Change/Increase & Total \\
\hline Non-preparation group & 1 & 7 & 5 & 13 \\
\hline Preparation group & 19 & 14 & 8 & 41 \\
\hline
\end{tabular}

Significant differences between groups (Chi-square test, $p<0.01$ )

Table 3: Improvement in bone defects at reevaluation. 
Citation: Sugaya T, Natatsuka M, Motoki Y, Inoue K, Tanaka S, et al. (2016) Sealing the Gap of Vertical Root Fracture through the Root Canal. Dentistry 6: 354 . doi:10.4172/2161-1122.1000354

limitations to treatment from within the root canal, making surgical treatment such as intentional replantation necessary.

Of the 41 teeth in the preparation group in which bone defects were seen prior to treatment, complete healing was achieved in 19 teeth (46.3\%) using nonsurgical treatment. It has been reported that if a tooth has already undergone root canal treatment and there is a bone defect at the apex, retreatment of the root canal has a success rate of $65.7 \%$ [25]. Removing bacteria from the fracture line and sealing the fracture gap is naturally more difficult than root canal treatment. Consequently, the success rate achieved in the present study may well be an appropriate outcome. The 1-2 month period may have been appropriate to clarify the improvement of probing depth and bone defects after preparing the fracture line through the root canal before bonding. However, the long-term follow-up results may include refracture and deterioration of the periodontal pocket and the effects of preparing and sealing of the fracture gap may vary. Further studies are needed to clarify the long-term prognosis and clinical significance. However, this method shows potential as a treatment for vertically fractured roots, because improvement was seen in many of the cases in the present study.

\section{Conclusion}

The results of the study suggest that preparing the fracture line through the root canal before sealing a vertically fractured root may be effective in resolving periodontal inflammation.

\section{References}

1. Polson AM (1977) Periodontal destruction associated with vertical root fractures. J Periodontol 48: 27-32.

2. Lommel TJ, Meister F, Gerstein H, Davies EE, Tilk MA (1978) Alveolar bone loss associated with vertical root fractures. Oral Surg Oral Med Oral Pathol 45: 909-919.

3. Pitts DL, Natkin E (1983) Diagnosis and treatment of vertical root fractures. J Endod 9: 338-346.

4. Barkhordar RA (1991) Treatment of vertical root fracture: a case report. Quintessence Int 22: 707-709.

5. Sugaya T, Kawanami M, Noguchi H, Kato H, Masaka N (2001) Periodontal healing after bonding treatment of vertical root fracture. Dent Traumatol 17: 174-179.

6. Kawai K, Masaka N (2002) Vertical root fracture treated by bonding fragments and rotational replantation. Dent Traumatol 18: 42-45.

7. Kudou Y, Kubota M (2003) Replantation with intentional rotation of a complete vertically fractured root using adhesive resin cement. Dent Traumatol 19: 115117

8. Hayashi M, Kinomoto Y, Takeshige F, Ebisu S (2004) Prognosis of intentional replantation of vertically fractured roots reconstructed with dentin-bonded resin. J Endod 30: 145-148.

9. Unver S, Onay EO, Ungor M (2011) Intentional re-plantation of a vertically fractured tooth repaired with an adhesive resin. Int Endod J 44: 1069-1078.

10. Kimura K, Sugaya T, Kato H (2000) Histopathological study of periodontal destruction after vertical root fractures. J Jpn Soc Periodontol 42: 255-266.

11. Tomita M, Sugaya T, Kawanami M (2002) Periodontal healing after intra-oral and extra-oral bonding treatment of experimental vertical root fracture. Jpn J Conserv Dent 45: 787-796.

12. Sugaya T, Nakatsuka M, Inoue K, Tanaka S, Miyaji H, et al. (2015) Comparison of fracture sites and post lengths in longitudinal root fractures. J Endod 41 : 159-163.

13. Noguchi $H$, Sugaya $T$, Kato $H$ (1997) Treatment of vertically fractured root by cohesion Part 2. Histological evaluation on cohesion with adhesin cement. Jpn J Conserv Dent 40: 1453-1460.

14. Taira Y, Imai $Y$ (2014) Review of methyl methacrylate (MMA)/tributylborane (TBB)-initiated resin adhesive to dentin. Dent Mater J 33: 291-304.

15. Listgarten MA, Mao R, Robinson PJ (1976) Periodontal probing and the relationship of the probe tip to periodontal tissues. J Periodontol 47: 511-513.

16. Armitage GC, Svanberg GK, Löe H (1977) Microscopic evaluation of clinical measurements of connective tissue attachment levels. J Clin Periodontol 4: 173-190.

17. Listgarten MA (1980) Periodontal probing: what does it mean? J Clin Periodontol 7: 165-176.

18. Tronstad L, Barnett F, Riso K, Slots J (1987) Extraradicular endodontic infections. Endod Dent Traumatol 3: 86-90.

19. Tronstad L, Kreshtool D, Barnett F (1990) Microbiological monitoring and results of treatment of extraradicular endodontic infection. Endod Dent Traumatol 6 : $129-136$.

20. Kiryu T, Hoshino E, Iwaku M (1994) Bacteria invading periapical cementum. J Endod 20: 169-172.

21. Lomçali G, Sen BH, Cankaya H (1996) Scanning electron microscopic observations of apical root surfaces of teeth with apical periodontitis. Endod Dent Traumatol 12: 70-76.

22. Noiri Y, Ehara A, Kawahara T, Takemura N, Ebisu S (2002) Participation of bacterial biofilms in refractory and chronic periapical periodontitis. J Endod 28: 679-683.

23. Leonardo MR, Rossi MA, Silva LA, Ito IY, Bonifácio KC (2002) EM evaluation of bacterial biofilm and microorganisms on the apical external root surface of human teeth. J Endod 28: 815-818.

24. Wang J, Jiang Y, Chen W, Zhu C, Liang J (2012) Bacterial flora and extraradicular biofilm associated with the apical segment of teeth with posttreatment apical periodontitis. J Endod 38: 954-959.

25. Ng YL, Mann V, Gulabivala K (2008) Outcome of secondary root cana treatment: a systematic review of the literature. Int Endod J 41: 1026-1046. 